\title{
Prototype of a radiation hard resistive bolometer for ITER
}

\author{
L.Giannone, D. Queen ${ }^{\dagger}$, F. Hellman ${ }^{\dagger}$ and J.C. Fuchs \\ Max Planck Institut fur Plasmaphysik, \\ EURATOM-IPP Association, D-85748 Garching, Germany \\ $\dagger$ University of California, Berkeley, CA 94270-7300, USA.
}

\section{Introduction}

A schematic diagram of the prototype radiation hard resistive bolometer foil is shown in Fig. 1. An isolating layer of silicon nitride is grown on a $500 \mu \mathrm{m}$ thick silicon wafer. This isolating layer of silicon nitride is sandwiched between a metal film layer that absorbs the radiation emitted from the plasma and a patterned meander track of metal. The temperature rise of the meander due to the absorbed radiation is measured by the change in resistance of the meander. The original bolometer foil used either a Kapton or mica isolation layer with a gold absorber and meander [1]. A reference and measurement absorber is used to compensate for the increase in meander resistance caused by an increase in bolometer head temperature during a discharge. The silicon frame supports the isolating layer of silicon nitride and provides the thermal contact to the bolometer head.

The original bolometer foil has been shown to perform unreliably in an environment with the high neutron fluences expected in ITER [2]. As the thermal neutron capture cross section of platinum is a factor 10 smaller than that of gold, it was suggested that the suspected transmutation problem experienced with the gold meander could be slowed down by substitution with platinum. Silicon nitride is currently being investigated as one of a number of ceramics to be used in the ITER environment [3]. A resistive bolometer with a platinum absorber and meander on a silicon nitride substrate could then be a suitable radiation hard bolometer detector for installation in ITER. Aspects of resistive metal bolometer foil design and possible alternatives for ITER have also been previously considered [4,5]

\section{Bolometer foil production}

Bolometer foil production parallels that of a similar device known as a microcalorimeter. Microcalorimeters have been operated up to $800 \mathrm{~K}$ [6] and in magnetic fields up to $8 \mathrm{~T}$ [7]. A silicon nitride film with 1.5 micron thickness is grown on a $500 \mu \mathrm{m}$ thick silicon wafer. The silicon nitride is grown by an LPCVD process ( low pressure chemical vapour deposition). Dichlorosilane $\left(\mathrm{SiH}_{2} \mathrm{Cl}_{2}\right)$ and ammonia are mixed at $835 \mathrm{C}$ to make a low stress film.

The platinum meander, with a thickness of $300 \mathrm{~nm}$ or $500 \mathrm{~nm}$, was then sputter deposited onto the silicon nitride film. The silicon was then etched away in the areas of dimension $4.3 \mathrm{~mm} \times 1.8 \mathrm{~mm}$ designated for the bolometer foil absorbers. An absorber layer with dimensions of $4 \mathrm{~mm} \times 1.5 \mathrm{~mm}$ was deposited with thicknesses of either $0.5,1.0$ or $1.5 \mu \mathrm{m}$ using sputtered deposition through a shadow mask. The prototype bolometer foils were produced at the Microfab facility of the University of California, Berkeley. A photograph of the bolometer foil viewed from the meander side is shown in Fig. 2. 


\section{Results}

The substitution of the prototype foils into existing bolometer heads with supporting cabling and electronics was facilitated by reproducing the exact dimensions of the present foils. An existing bolometer head using a prototype foil was installed in ASDEX Upgrade. In Fig. 3, the time evolution of the bolometer signals in a discharge at $2.5 \mathrm{~T}$ with a plasma current of $800 \mathrm{kA}$ and $5 \mathrm{MW}$ neutral beam heating is plotted. The power flux incident onto each absorber viewed along the four tangential lines of sight can be calculated from these signals. Four months of continuous operation without failure indicate that the prototype bolometer foil is robust enough for installation in a tokamak.

In Fig. 4, the results of simulations of various bolometer foils are shown. Using the specific heat, density and thermal conductivity of Kapton and gold, the time evolution of the mean temperature of the absorber of the original bolometer foil (green) was calculated. Using those values for low stress silicon nitride and platinum, the time evolution of the mean temperature of the absorber of the bolometer foil (purple) with $0.15 \mathrm{~mm}$ between the absorber edge and silicon frame was calculated. In both cases it is assumed that a source of $40 \mathrm{~W} / \mathrm{m}^{2}$ is switched on at $t=0 \mathrm{~s}$. The time evolution of the mean temperature of the absorber for bolometers on a silicon nitride membrane with a $4 \mu \mathrm{m}$ (red) and $12 \mu \mathrm{m}$ (orange) Pt absorber assuming a $0.5 \mathrm{~mm}$ separation between absorber and silicon frame and a bolometer with a $20 \mu \mathrm{m}$ thick mica isolator and $4 \mu \mathrm{m}$ gold absorber (blue) is also shown.

The experimental values of heat capacity at $50 \mathrm{C}$ are $40 \mu \mathrm{J} / \mathrm{K}$ for the prototype and $160 \mu \mathrm{J} / \mathrm{K}$ for the original bolometer. The cooling time constant is $60 \mathrm{~ms}$ for the prototype bolometer and $110 \mathrm{~ms}$ for the original bolometer. As the 2D heat diffusion simulations use only the nominal values of the component dimensions and the literature values for their specific heat, density and thermal conductivity [9,10,11,12], only a qualitative agreement could be expected with the measured heat capacity and cooling time constant. Despite these qualifications, the measured and calculated values of heat capacity and cooling time constant are in agreement to within $15 \%$. This confirms that the performance of future designs can be predicted with adequate precision.

Increasing the distance of the absorber to the silicon frame to $0.5 \mathrm{~mm}$ will increase the cooling time constant to $227 \mathrm{~ms}$ and increase the sensitivity of foils with a $4 \mu \mathrm{m}$ Pt absorber by a factor of 3.6 compared to the currently used Kapton and gold bolometer foil with a $4 \mu \mathrm{m}$ absorber. It is projected that the ITER bolometer with a $12 \mu \mathrm{m}$ thick platinum absorber would have a cooling time constant of $225 \mathrm{~ms}$ and a factor of 1.5 improvement in sensitivity compared to the currently used Kapton and gold bolometer foil. To produce bolometer foils with the lowest possible thermal drift necessary for ITER long pulse operation, it will be necessary to better match the heat capacity, cooling time constant and meander resistances of the measurement and reference foil.

\section{Discussion}

The calibration of bolometer foils as a function of temperature reveals that the normalised heat capacity, $\kappa$ and cooling time constant, $\tau$ increase with temperature. This feature can be explained in terms of the increase in specific heat of the component materials with temperature. This effect dominates the increase in thermal conductivity with temperature which would tend to lead to a decrease in $\tau$ with increasing temperature. This temperature dependence of the calibration constants must be accounted for when operating a bolometer camera to obtain a reliable tomographic inversion of the measured line integrals of power flux.

Further improvements to the prototype bolometer foil will be the aim of the next round 


\begin{tabular}{|c||c|c|c|}
\hline Metal & Neutron capture cross section (barns) & $\rho(\mu \Omega . c m)$ & TCR $\left(\mathrm{K}^{-1}\right)$ \\
\hline \hline $\mathrm{Au}$ & 98.7 & 2.2 & 0.0034 \\
\hline $\mathrm{Pt}$ & 10.3 & 10.6 & 0.0035 \\
\hline $\mathrm{Ni}$ & 4.4 & 6.9 & 0.0061 \\
\hline $\mathrm{W}$ & 18.4 & 5.5 & 0.0041 \\
\hline
\end{tabular}

Table 1: A comparison of the neutron capture cross section, resistivity at $\mathrm{T}=20 \mathrm{C}$ and temperature coefficient of resistivity, TCR, of candidate metals for the bolometer meander.

of foil production. The prototype foil will have an absorber thickness close to that required for ITER operation. A prototype bolometer foil with a nickel meander is proposed, since this material has a smaller thermal neutron capture cross section than platinum and would increase the bolometer foil life time in ITER (see Table 1). With a nickel meander the bolometer sensitivity would also be increased, as the temperature coefficient of resistivity of nickel is larger than that of platinum. However, the operating temperature of the bolometer foil must be less than $550 \mathrm{~K}$, as above this temperature a ferromagnetic transition in nickel results in a decrease of the temperature coefficient of resistivity.

The actual absorber thickness to use on ITER remains to be established. This requires the examination of the various ITER scenarios and their expected radiation profiles and energy spectra to ascertain whether a given absorber thickness produces an unacceptable measurement of the total radiated power. An in situ test of the prototype bolometer foil in the presence of an ITER relevant neutron fluence to demonstrate the radiation hardness of low stress silicon nitride is planned. Development work is also needed to establish a radiation hard method of contacting the cable to the foil.

It is necessary to assess whether the power flux produced in an ITER disruption will cause an intolerable temperature rise of the absorber and damage the bolometer foil.

\section{References}

[1] K. Mast et al., Rev. Sci. Instrum. 62, 744 (1991)

[2] R. Reichle et al., in 28th European Conference on Controlled Fusion and Plasma Physics (Madiera), volume 25A, page 1293, 2001.

[3] M. González and E. Hodgson, Fusion Eng. Design 66-68, 881 (2003)

[4] R. Reichle et al., in Diagnostics for experimental thermonuclear fusion reactors, edited by P. Stott et. al, page 559, Plenum Press, New York, 1996.

[5] R. Reichle, M. Di Maio, and L. Ingesson, in Diagnostics for experimental thermonuclear fusion reactors 2, edited by P. Stott et. al, page 389, Plenum Press, New York, 1998.

[6] D. Denlinger et al., Rev. Sci. Instrum. 65, 946 (1994).

[7] B. Zink, B. Revaz, R. Sappey, and F. Hellman, Rev. Sci. Instrum. 73, 1841 (2002).

[8] L. Giannone, K. Mast, and M. Schubert, Rev. Sci. Instrum. 73, 3205 (2002).

[9] C. Mastrangelo, Y. Tai, and R. Muller, Sensors and Actuators A21-A23, 856 (1990).

[10] B. Revaz et al., Rev. Sci. Instrum. 74, 4389 (2003).

[11] R. Weast, editor, Handbook of Chemistry and Physics, CRC Press, Cleveland, Ohio, USA, 1970.

[12] Data sheet, Physical and thermal properties of Kapton, http://www.dupont.com/kapton/general/spphythe.html, 2005. 


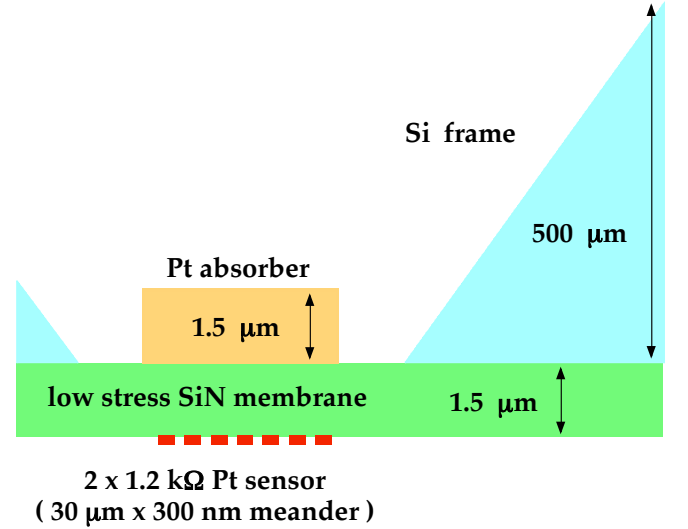

Figure 1: Schematic diagram of a prototype bolometer foil. An isolating layer of low stress silicon nitride (green) with a Pt meander (red) and Pt absorber (orange) is supported by a silicon frame (light blue).

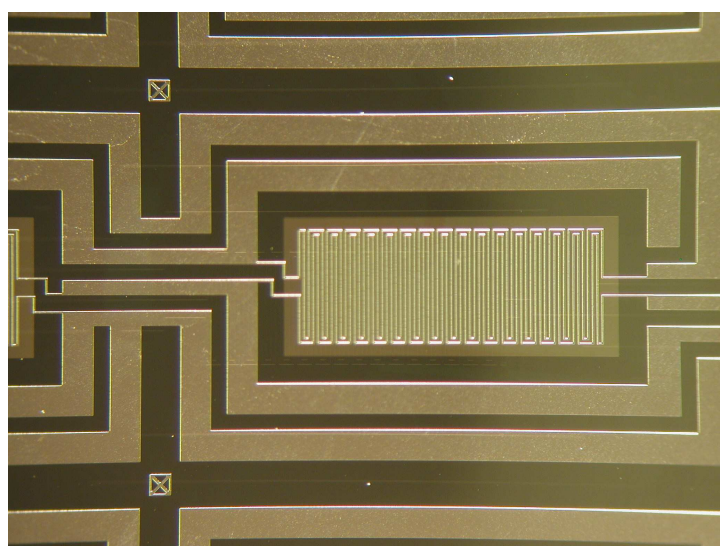

Figure 2: Bolometer foil overview showing one pair of meanders and the interconnections between the reference and measurement foil. The meander width is $30 \mu \mathrm{m}$ and the thickness is either $300 \mathrm{~nm}$ or $500 \mathrm{~nm}$.

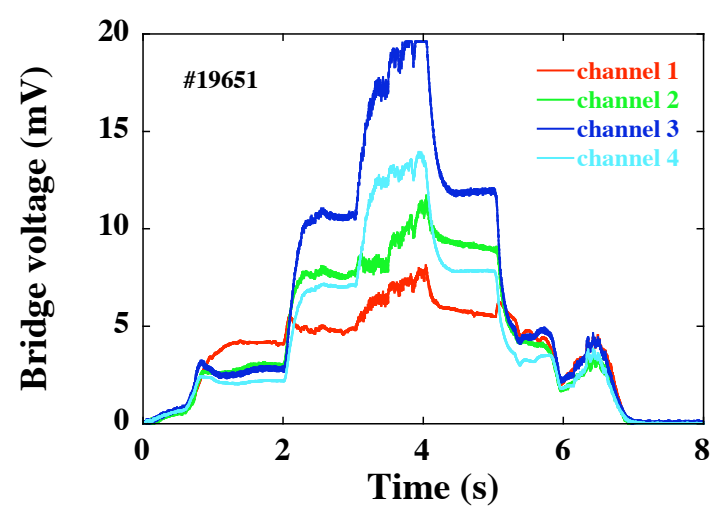

Figure 3: Time evolution of signals from the prototype bolometer in a discharge at $2.5 \mathrm{~T}$ with $I_{p}=800 \mathrm{kA}$ and $5 \mathrm{MW}$ neutral beam heating.

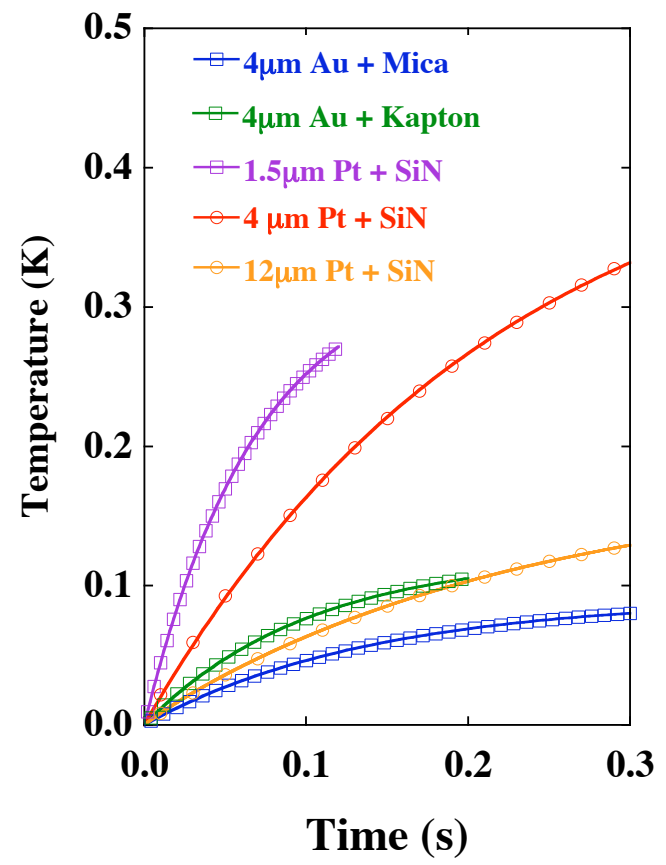

Figure 4: The simulated time evolution of the mean temperature of the absorber when switching on a $40 \mathrm{~W} / \mathrm{m}^{2}$ source at $t=0 \mathrm{~s}$ is plotted for various bolometer foils. 